\title{
Marco institucional para la gestión ambiental en el Perú
}

\author{
Institutional framework for environmental management \\ in Peru
}

WALTER VALDEZ MUÑOZ*

\begin{abstract}
Resumen: El presente artículo desarrolla el proceso de inclusión de la gestión ambiental como una obligación del Estado, presentando el contexto en el que se da esa inclusión, su evolución en América Latina y el Caribe. Para culminar se discute lo ocurrido en el caso peruano y se plantea algunas propuestas que desde la consideración del autor podrían mejorar la institucionalidad ambiental en el Perú.
\end{abstract}

Palabras clave: gestión ambiental - institucionalidad ambiental - obligaciones estatales

Summary: The unfolding inclusion process of environmental management as a Government obligation is developed in this article, explaining its context and evolution in Latin America and the Caribbean plus the Peruvian case. The present document also sets some proposals, according to the author point view, for improvement of the environmental institutional structure in Peru.

Key words: environmental management - environmental institutional structure - governmental obligations

CONTENIDO: I. INTRODUCCIÓN.- II. LA INCORPORACIÓN DE LA DIMENSIÓN AMBIENTAL COMO FUNCIÓN DEL ESTADO.- III. LA EVOLUCIÓN DE LOS MARCOS INSTITUCIONALES EN AMÉRICA LATINA.- III.1. MINISTERIOS O AGENCIAS SECTORIALES.- III.2. MINISTERIOS O AGENCIAS DE UN SECTOR SOCIAL CON ATRIBUCIONES DE CARÁCTER AMBIENTAL.- III.3. CREACIÓN DE MINISTERIOS O UNIDADES A PARTIR DE REFORMAS PARA EL LOGRO DE ESTRATEGIAS DE PLANEAMIENTO TERRITORIAL Y UNA PLANIFICACIÓN REGIONAL.- III.4. MINISTERIOS O UNIDADES AUTÓNOMOS DEL AMBIENTE.III.4.1. CREACIÓN DE UN MINISTERIO DEL AMBIENTE.- III.4.2. CREACIÓN DE UN ORGANISMO AMBIENTAL AD-HOC QUE NO TIENE EL RANGO DE MINISTERIO Y QUE SE UBICA GENERALMENTE DEBAJO DEL JEFE DE GOBIERNO O DE LOS ÓRGANOS DE LA PLANIFICACIÓN.- III.5. CREACIÓN DE OFICINAS AMBIENTALES DENTRO DE MINISTERIOS DE ECONOMÍA O UNIDADES DE PLANIFICACIÓN DE ALTO PESO POLÍTICO.- III.6. CREACIÓN DE COMISIONES EJECUTIVAS O AGENCIAS CENTRALES DEPENDIENTES DE LA PRESIDENCIA DE LA REPÚBLICA O DEL MÁS ALTO NIVEL DEL GOBIERNO

\footnotetext{
* Abogado por la Pontificia Universidad Católica del Perú (PUCP), con un posgrado en Gobernabilidad y Gerencia Política por la George Washington University y la PUCP, egresado de la Maestría en Gerencia Social de la PUCP, autor de diversas publicaciones en temas ambientales. Correo electrónico: wvaldez@estudiovaldezmunoz.com
} 
DE CARÁCTER INTERSECTORIAL.- III.7. CREACIÓN DE SUPERAGENCIAS

O COMISIONES INTERSECTORIALES COMBINADAS CON UNIDADES CENTRALES Y DESCENTRALIZACIÓN EN LOS NIVELES REGIONALES $Y$ LOCALES.- III.8. CREACIÓN DE UN SISTEMA NACIONAL DEL AMBIENTE.IV. EL MARCO JURÍDICO INSTITUCIONAL AMBIENTAL EN EL PERÚ.-V. ANEXO.

\section{INTRODUCCIÓN}

Toda actividad económica que involucra extracción de recursos naturales (pesquera, forestal, energética, minera, etcétera) genera impactos ambientales, no solo en el proceso extractivo, sino también en el tratamiento y transformación de los recursos extraídos. Del deterioro ambiental actual no cabe duda alguna, así como que todos los habitantes compartimos el mismo planeta y que el deterioro ambiental no conoce de límites políticos, económicos o geográficos. Y así como se habla de globalización en términos económicos también debemos reconocer que vivimos en un mundo interdependiente ambientalmente hablando. Adicionalmente compartimos la obligación de legar un ambiente adecuado a las futuras generaciones.

Si bien es cierto que en la actualidad tenemos identificados una serie de problemas ambientales como el calentamiento global, el deterioro de la capa de ozono, la destrucción de bosques tropicales y otros ecosistemas, con la consiguiente pérdida de especies, la contaminación y en muchos casos el agotamiento del agua dulce, etcétera; lo más preocupante es que carecemos de información sobre los efectos tóxicos o de otra índole que producen el ingreso de una serie de productos sintéticos y químicos al medio natural. Por otra parte, tampoco estamos seguros si los científicos desarrolladores de nuevas técnicas biogenéticas tienen procedimientos efectivos, seguros y controlados para evitar daños graves en el futuro. Es decir trabajamos en desarrollar nuevas tecnologías para producir más, pero no hacemos mucho para eliminar de manera segura los desperdicios o controlar los riesgos de las nuevas tecnologías. Respecto a la extracción de recursos naturales, no solo son un problema los impactos de la extracción de los mismos del medio natural, sino que en casos como la pesquería (aunque en la actualidad el problema más grave es el de la sobrepesca) ha sido durante mucho tiempo el procesamiento y transformación del recurso lo que ha generado graves problemas de contaminación. Esto también ocurre con los minerales que, a decir de Mario Valls, «no tanto la extracción de minerales, sino más bien los procesos consiguientes de concentración, fundición y refinación, cuya integración local o regional con las explotaciones es estimulada, pueden afectar el medio ambiente» ${ }^{1}$. Claro que en el caso peruano en

1 Valls, Mario Francisco. Manual de Derecho Ambiental. Buenos Aires: Ugerman, 2001. 
la actualidad, los mayores problemas de la actividad minera se vienen dando por la disputa del recurso suelo y el uso del recurso agua, recursos de uso múltiple, fundamentalmente en áreas comunales de la zona andina.

En el Perú, debemos reconocer que el desarrollo de las actividades productivas como la minería ha sido durante muchos años poco o nada amigable con el medio ambiente, al punto que aun después de veinte años del establecimiento normativo de una serie de exigencias ambientales, el mayor número de conflictos en el país son conflictos a los que se ha venido en denominar conflictos socioambientales. Según la Defensoría del Pueblo existían en el Perú hasta febrero de 2013, 147 conflictos socioambientales ${ }^{2}$.

El deterioro ambiental en el mundo nos lleva a constatar que este es mucho más veloz que la maduración de la conciencia colectiva ${ }^{3}$ y, por lo tanto, surge la necesidad de frenarlo mediante la regulación de conductas (el Derecho) y la creación de una institucionalidad desde el Estado4. Según el Informe Planeta Vivo correspondiente al año 20125, preparado por el World Wide Life Fund for Nature (WWF), ya en el año 2008 se había producido un desfase anual de 0,5 entre la demanda de recursos naturales renovables por parte de la población mundial y la oferta de ellos por parte de la naturaleza, es decir que la tierra requería 1,5 años para regenerar los recursos naturales renovables utilizados por la población mundial en un año. Este panorama requiere pues de una respuesta «contundente y sólida» ${ }^{6}$ por parte del Estado, es decir, una institucionalidad coherente no solo en su ubicación dentro del aparato estatal, sino que además debe contar con los recursos humanos, técnicos y financieros necesarios.

\section{LA INCORPORACIÓN DE LA DIMENSIÓN AMBIENTAL COMO FUNCIÓN DEL ESTADO}

Frente al panorama ambiental descrito, se hizo necesario establecer un marco jurídico que permita el aprovechamiento de los recursos naturales sin afectar de manera dramática el medio ambiente. La incorporación de estas exigencias ambientales como una obligación de los Estados se afianza en el marco de la Conferencia sobre el Medio Humano del año 1972, conocida como Conferencia de Estocolmo por el lugar en que se llevó a cabo. Como parte de los resultados de la Conferencia se reconoce

2 Defensoría del Pueblo. Reporte de Conflictos Sociales, X, 108 (2013).

3 Ver, Tudela, Fernando. Hacia un nuevo pacto internacional para el desarrollo sustentable. Perspectivas de América Latina y el Caribe. Banco Interamericano de Desarollo, 1992.

4 Ibídem.

5 WORLD WIDE LIFE FUND FOR NATURE (WWF). Planeta vivo. Informe 2012. Biodiversidad, biocapacidad y propuestas de futuro. Gland, Suiza: www International, p. 38.

6 MonRoy Rosas, Juan Carlos. El costo de la indiferencia ambiental: valoración de costos ambientales, instrumentos de política y crisis ambiental. Bogotá: Editorial Universidad del Rosario, p. 89.

MARCO

INSTITUCIONAL PARA LA GESTIÓN AMBIENTAL EN EL PERÚ

INSTITUTIONAL FRAMEWORK FOR ENVIRONMENTAL MANAGEMENT IN PERU 
no solo la existencia de problemas ambientales serios, sino que se adopta un Plan de Acción que compromete a los Estados miembros de las Naciones Unidas a asumir como una obligación del Estado el cuidado del medio ambiente. Definitivamente el deterioro ambiental no podía seguir asimilándose como un costo aceptable del desarrollo?

También los organismos financieros multilaterales como el Banco Mundial, los bancos regionales como el Banco Interamericano de Desarrollo (BID) y las agencias de cooperación se comprometieron a contribuir a que los países miembros de esas instituciones cumplan una serie de exigencias respecto a los proyectos financiados por estos organismos financieros. Se comprometieron también a ayudar a los países a enfrentar los problemas ambientales y lograr un aprovechamiento de sus recursos naturales de manera sostenible ${ }^{8}$.

El Estado es quien impone límites al ejercicio de los derechos, es un ente regulador de conductas $^{9}$, y al reconocer los Estados que los problemas ambientales son problemas que afectan a la sociedad en su conjunto, la protección del medio ambiente, a partir de los años setenta, se torna en una responsabilidad de la administración pública. La conservación del medio ambiente como una función del Estado, lo habilita y lo obliga, al mismo tiempo, a intervenir en sus distintos niveles de gobierno en la gestión ambiental, más aún cuando hay la percepción de que el ambiente es un bien colectivo. Es en este escenario que los Estados comienzan a modificar sus estructuras administrativas y sus ordenamientos jurídicos para hacer frente a los problemas ambientales, especialmente considerando que en algunos casos los recursos naturales no solo no son renovables, sino que en caso lo fueran, se requeriría de ciertas condiciones difíciles de alcanzar sin la intervención del Estado. La autorregulación no es posible en un escenario donde por mucho tiempo se ha apostado por un modelo de desregulación ${ }^{10}$ y donde los propios actores desconocen los efectos futuros de su desempeño respecto de la naturaleza. La problemática ambiental es compleja y los decisores políticos deben tener en cuenta que la solución de los problemas requiere del aporte de la comunidad científica y que la solución del problema no se circunscribe a un solo ámbito de estudio. De allí que es imprescindible un Estado con la suficiente capacidad profesional para fundamentar debidamente sus decisiones y con autoridad sobre los ciudadanos y las empresas para enfrentar el deterioro ambiental.

7 BARCENA IBARRA, Alicia. Reflexiones sobre la incorporación de la dimensión ambiental en el marco institucional y operativo del sector público en América Latina y el Caribe. Washington: BID, 1987.

8 SzeKely, Francisco. Estrategias para el Fortalecimiento de la Calidad Ambiental en el ciclo de Proyectos de Desarrollo del BID. Washington: BID, 1987, p. 87.

9 FeRnández de GatTA SÁnCHeZ, Dionisio. Sistema Jurídico Administrativo para la Protección del Medio Ambiente. Salamanca: Ratio Legis, 2012.

10 MESA CuADROS, Gregorio. «Principios ambientales como reglas de organización para el cuidado, la vida, la conservación y el futuro". En MESA CuADROS, Gregorio (org.). Debates ambientales contemporáneos. Bogotá: Universidad Nacional de Colombia, 2010. 
La incorporación a la administración pública de recursos humanos, técnicos y financieros y una institucionalidad fuerte para mejorar la gestión ambiental es fundamental. No es posible alcanzar un desarrollo sostenible sin inversión económica, sin capacitación permanente a los funcionarios públicos, se requiere pues de una formación sólida de los funcionarios públicos desde el punto de vista cognoscitivo, afectivo y ético. En la actualidad los Estados tienen claro que no solo pueden ejercer el poder de policía así como su potestad de inspección y sanción, sino que también pueden fomentar ciertas prácticas amigables con el medio ambiente, establecer estímulos que pueden ser de orden tributario u otros instrumentos de mercado.

\section{LA EVOLUCIÓN DE LOS MARCOS INSTITUCIONALES EN AMÉRICA LATINA}

La situación ambiental de América Latina y el Caribe llevó a los Estados a buscar mecanismos de gestión ambiental que les permitan enfrentar básicamente dos aspectos. El primero de estos aspectos es la intervención en los espacios territoriales en los que la capacidad asimiladora y regeneradora de la naturaleza ha sido rebasada. Espacios cuya recuperación solo era posible a través del desarrollo de acciones estatales ambientales adecuadas. El segundo es la incorporación de criterios ambientales en la planificación del desarrollo nacional con la finalidad de anticiparse a y evitar acontecimientos económicos, sociales y ambientales de gran impacto, en lugar de simplemente reaccionar a ellos.

El segundo de estos dos aspectos es especialmente importante para los países de la región que sufren de escasez de los recursos técnicos y económicos necesarios para cubrir los elevados y repetitivos costos ambientales de la reversión de situaciones de desequilibrio ecológico. Situaciones provocadas principalmente por la carencia de una planificación y previsión de los efectos ambientales. Lo que debe quedar claro para nuestros países es que, si bien es cierto que una política de este tipo incurre en costos de planeamiento, de investigación y de acción preventiva, y a veces en retrasos y modificaciones de determinados proyectos de desarrollo, en el largo plazo las ventajas son mayores, pues se evitan errores que pueden hacer fracasar los esfuerzos del desarrollo, limitarlo y causar mayores gastos a países carentes de recursos.

Al quedar más o menos definido el accionar de los Estados, accionar que implicaba asumir una gestión pública del medio ambiente y los recursos naturales, se presentaba la necesidad de contar con una estructura jurídico administrativa que les permitiera cumplir de manera apropiada su función ambiental. Esto significaba una transformación de sus estructuras, consistentes básicamente en la apertura de espacios administrativos encargados de la gestión ambiental. 
El proceso de incorporación de la dimensión ambiental en las estructuras de los países de América Latina se inició a mediados de la década de 1970 con la incorporación de ciertas funciones ambientales en sectores vinculados a la gestión de recursos naturales como la agricultura, la pesca, la minería y en algunos casos a sectores sociales como el sector salud o de infraestructura como vivienda y saneamiento. Es decir se incorporó la dimensión ambiental al Estado de manera sectorializada. Un segundo paso fue la incorporación de la dimensión ambiental en el proceso de planificación del desarrollo. Sin embargo, en muchos casos la planificación no tenía un engarce con quienes manejaban la economía, por lo tanto, esos planes de desarrollo que incorporaron la visión ambiental no pasaron de ser una expresión de deseos. Un tercer momento fue la creación de Comisiones o Consejos Multisectoriales con la participación de sectores productivos y sociales con la pretensión de darle una mayor transversalidad a la gestión ambiental. El rol de estas comisiones era fundamentalmente de coordinación ${ }^{11}$. Sin embargo, excepto por casos como el de Chile donde el CONAMA (Comisión Nacional del Medio Ambiente) sí funcionó, en la mayoría de países estos intentos fracasaron por la debilidad de las instituciones que los respaldaban. En la actualidad, los países de América Latina y el Caribe han optado mayoritariamente por la creación de un ministerio. Cada uno de los modelos adoptados por los países de la región a lo largo de estos años ofrece ventajas y desventajas ${ }^{12}$.

\section{III.1. Ministerios o agencias sectoriales}

En la mayoría de casos de ministerios o agencias sectoriales, se trataba de sectores productivos tradicionales como agricultura, industrias, minería o pesquería, a los cuales se les asignaba adicionalmente atribuciones para conducir e instrumentar políticas ambientales. Este modelo de gestión ambiental presenta muchos inconvenientes, ya que estos sectores generalmente tienen intereses, experiencia técnica e información adecuadas para el cumplimiento de sus actividades productivas sectoriales. Adicionalmente aparece como incompatible su rol prioritariamente productivo con sus competencias ambientales si se tiene en cuenta que los recursos naturales son insumos esenciales en las actividades productivas. Asimismo existen otros cuestionamientos a este modelo:

- Conflicto con el organismo central. Esto es frecuente en países donde hay organismos centrales de política y control ambiental y adicionalmente Ministerios que tratan sectorialmente el

11 EsPINOZA, Guillermo y Manuel RodríGueZ. Gestión ambiental en América Latina y el Caribe: Evolución, tendencias y principales prácticas. Washington: BID, Departamento de Desarrollo Sostenible, División de Medio Ambiente, 2002.

12 VALDEZ MUÑOZ, Walter. La función del Estado y el Derecho en la solución de los problemas ecológicos. El caso peruano. Tesis de bachillerato. Lima: PUCP, 1990. 
problema, generándose en muchos casos conflictos entre ambas instancias.

- Duplicación de funciones con el organismo central. Se producen mayores gastos, al duplicar acciones para la consecución de un mismo fin.

- Es juez y parte de su gestión ambiental. Los sectores de la producción son los que a través de sus actividades tienen una mayor incidencia ambiental y los que utilizan como insumos esenciales recursos naturales.

- Cuando es responsable del control de la gestión de otros sectores, es frecuente encontrar en su acción normativa limitaciones para influir en la acción de otras entidades.

Por otro lado, un aspecto positivo que podemos rescatar del desarrollo de una experiencia de este tipo ha sido que los ministerios productivos se han visto obligados a incorporar profesionales con una formación ambiental para que se ocupen de las nuevas tareas asociadas al cumplimiento de sus funciones ambientales. Consideramos que esto coadyuva al logro de una disciplina ambiental, por lo menos en teoría. En la región se asignaron competencias a sectores productivos competencias ambientales, ministerios como los de agricultura, minas, industrias.

\section{III.2. Ministerios o agencias de un sector social con atribuciones de carácter ambiental}

La variación de este modelo respecto al anterior radica en que aquí la nueva unidad se crea como parte de un ministerio o agencia del sector social, salud, vivienda y en las áreas relativas a ciencia y tecnología. Esto se explica por la relación existente entre problemática ambiental y la carencia de servicios básicos y problemas tecnológicos. Principalmente se crearon unidades en el sector salud para atender los problemas de la contaminación de la salud humana, permaneciendo el manejo de los recursos naturales renovables y no renovables en sectores productivos como agricultura, energía, minería, etcétera.

$\mathrm{Al}$ igual que en el modelo anterior, en casi todos los países de la región se establecieron en el Ministerio de Salud unidades de saneamiento ambiental. Una crítica a este modelo es su enfoque antropocéntrico del problema y la visión sectorial que asume de la gestión ambiental. 


\section{III.3. Creación de ministerios o unidades a partir de refor- mas para el logro de estrategias de planeamiento territorial y una planificación regional}

Este modelo está fundamentalmente referido al desarrollo urbano y se explica por el acelerado proceso de urbanización e industrialización de las últimas décadas del siglo pasado. Se crearon ministerios de vivienda y en los casos en que ya existían se inauguraron unidades de planificación ambiental para hacer frente a la demanda de recursos de los sectores primarios, demanda de servicios urbanos y a la generación de residuos de los procesos productivos generadores de graves problemas de contaminación. Un esquema de este tipo es eminentemente operativo y tiene como objetivo principal compatibilizar todas las acciones y servicios urbanos a nivel regional. Sin embargo, tiene un accionar restringido.

\section{III.4. Ministerios o unidades autónomos del ambiente}

En muchos países se establecieron unidades especializadas en materia ambiental y recursos naturales. Dichas unidades tenían la función de asesorar a los gobernantes acerca de las políticas de gestión sobre la materia, coordinar las actividades con diversos organismos y manejar las relaciones internacionales del país en aspectos ambientales.

\section{III.4.1. Creación de un ministerio del ambiente}

Este esquema podía presentar hasta tres maneras de asumir las competencias ambientales:

- Un superministerio en el que se integran todos los ministerios con competencias ambientales importantes.

- Un ministerio especializado en el que se integran solo aquellas unidades con competencias ambientales específicas.

- Un ministerio puramente coordinador, es decir, que el nuevo ministerio coexiste con las estructuras jurídico-administrativas anteriores que mantienen sus competencias ambientales específicas y se limita a coordinar acciones.

III.4.2. Creación de un organismo ambiental ad-hoc que no tiene el rango de ministerio y que se ubica generalmente debajo del Jefe de Gobierno o de los órganos de la planificación

Estos organismos pretendían ser una instancia que globalice la política ambiental y tener funciones de ejecución y/o control de las mismas políticas. Las ventajas que ofrece este modelo son las siguientes:

- formar cuadros profesionales en el campo ambiental; 
- concentrar una gran cantidad de funciones dispersas en el sector público;

- incrementar la asignación de recursos y particularmente la capacidad de gestionar ante las instituciones financieras, créditos y estímulos e incentivos económicos para obras de saneamiento básico o control de la contaminación ambiental;

- mayor participación de la industria privada frente a la existencia de un sector que ejerce mucha presión en lo normativo con el apoyo de la legislación vigente, o en lo concertado, con el apoyo de incentivos fiscales o crediticios;

- posibilidad de interactuar más eficazmente con el sector social, principalmente con las organizaciones no gubernamentales que son un vínculo esencial entre el gobierno y la población;

- facilitar la coordinación de las relaciones internacionales en materia ambiental;

- se convierte en un centro de imputación interna a la que recurren las personas naturales o jurídicas frente a cualquier problema ambiental.

Entre las desventajas que presenta este esquema podemos señalar las siguientes:

- en el mediano o largo plazo hay una tendencia a marginar la dimensión ambiental de la toma de decisiones y de la planificación del desarrollo;

- entre las unidades centrales y los organismos sectoriales surgen conflictos, debido a la evidente contraposición entre el interés por el crecimiento económico y el referente a la protección ambiental;

- podría crear un esquema de subordinación no recomendable por su rigidez y concentración, ya que en materia ambiental se requiere de flexibilidad para aquellas funciones transectoriales que exigen una relación más horizontal.

\section{III.5. Creación de oficinas ambientales dentro de ministerios de economía o unidades de planificación de alto peso político}

En virtud de este sistema, las propuestas relacionadas con el medio ambiente se sitúan en un nivel próximo al del planeamiento económico nacional. Se puede reconocer como una ventaja de este modelo la vinculación de las oficinas ambientales con unidades de alta autoridad política. Esto permite que dichas oficinas cuenten con el apoyo de todos los sectores del gobierno para cualquier iniciativa. Adicionalmente 
esta variante permite la incorporación de expertos ambientales en los consejos y unidades nacionales de planificación económica.

En estos casos las unidades ambientales pueden funcionar como agencias, secretariados o institutos descentralizados dentro del ministerio de planificación, de economía o dentro de la oficina del primer ministro. Si tuviéramos que hablar de las ventajas y desventajas de este modelo, podríamos decir que parece ser sumamente eficaz en relación con el dictado de normas y reglamentos, el ejercicio de influencia política a nivel nacional, la coordinación con otros sectores y el ejercicio del poder político, pero es ineficiente a los efectos de resolver controversias entre distintos organismos e influir decididamente sobre el medio ambiente local.

\section{III.6. Creación de comisiones ejecutivas o agencias centrales dependientes de la Presidencia de la República o del más alto nivel del gobierno de carácter intersectorial}

Este modelo resulta interesante por el alto peso político que le da a las decisiones y por el carácter participativo en los diferentes sectores del gobierno. Estas comisiones pueden perfectamente combinarse con otras unidades de carácter sectorial.

\section{III.7.Creación de superagencias o comisiones intersectoriales combinadas con unidades centrales y descentralización en los niveles regionales y locales}

Este esquema no está constituido por oficinas ambientales propiamente dichas, sino por organismos especiales, cada uno de los cuales tiene a su cargo la totalidad de una región geográfica determinada. Un caso típico de este modelo es la gestión del territorio organizado bajo determinados ejes geográficos como puede serlo una cuenca.

Este esquema puede considerarse de suma eficacia en lo concerniente a influir en el medio ambiente local y establecer directrices y normas para regiones específicas. Es ineficaz en lo que se refiere al logro del objetivo de incorporar la planificación ambiental en el proceso de desarrollo a nivel nacional.

\section{III.8. Creación de un sistema nacional del ambiente}

Este modelo consiste en la creación de una organización funcional en la que el conjunto de organismos públicos se relacionan entre sí en razón de las funciones de carácter ambiental que están llamados a cumplir. Este 
esquema teóricamente aparece como adecuado para resolver de una manera apropiada los problemas de centralización y descentralización, de sectorización y de globalización entre otros. Las ventajas que ofrece tener un ministerio del ambiente es que permite lo siguiente:

- formar cuadros profesionales en el campo ambiental;

- concentrar una gran cantidad de funciones dispersas en el sector público;

- incrementar la asignación de recursos y particularmente la capacidad de gestionar ante las instituciones financieras, créditos y estímulos e incentivos económicos para mejorar la gestión ambiental;

- mayor participación de los sectores productivos frente a la existencia de un sector que ejerce mucha presión en lo normativo con el apoyo de la legislación vigente, mediante la fiscalización y sanción o en lo concertado, con el apoyo de incentivos fiscales o crediticios

- interactuar más eficazmente con el sector social, principalmente con las organizaciones no gubernamentales que son un vínculo esencial entre el gobierno y la población;

- facilitar la coordinación de las relaciones internacionales en materia ambiental;

- se convierte en un centro de imputación interna al que recurren las personas naturales o jurídicas frente a cualquier problema ambiental.

Sin embargo, el modelo también presenta desventajas que no habría que perder de vista:

- en el mediano o largo plazo, hay una tendencia a marginar la dimensión ambiental de la toma de decisiones y de la planificación del desarrollo;

- entre las unidades centrales y los organismos sectoriales surgen conflictos, debido a la evidente contraposición entre el interés por el crecimiento económico y el referente a la protección ambiental;

- podría crear un esquema de subordinación no recomendable por su rigidez y concentración, ya que en materia ambiental se requiere de flexibilidad para aquellas funciones transectoriales que exigen una relación más horizontal.

Respecto a mantener competencias ambientales en sectores productivos también hay argumentos de ambos lados. Como argumentos en contra tenemos la posibilidad de que las competencias queden en los sectores

MARCO

INSTITUCIONAL

PARA LA GESTIÓN

AMBIENTAL EN EL

PERÚ

INSTITUTIONAL

FRAMEWORK FOR

ENVIRONMENTAL

MANAGEMENT IN

PERU 
productivos tradicionales (minería, energía, agricultura, pesquería, etcétera), pues tienen interés, experiencia técnica e información adecuada para el cumplimiento de sus actividades productivas sectoriales. Y en segundo lugar, sabemos que se actúa como juez y parte de la gestión ambiental, toda vez que son los sectores de la producción los que a través de sus actividades tienen una mayor incidencia ambiental y utilizan como insumos esenciales recursos naturales. Como argumentos a favor, consideramos que existe un argumento que bien valdría la pena tener en cuenta y es que la asignación de atribuciones a los sectores productivos para producir e instrumentar políticas ambientales los ha llevado no solo a abrir oficinas encargadas de tales funciones, sino que han tenido que incorporar profesionales que tengan una formación mínima en materia ambiental.

Esto último podría conducirnos en el mediano plazo al logro de una disciplina ambiental en los sectores productivos de tal modo que el éxito de la gestión ministerial no se mida en volúmenes de producción, sino en términos económicos reales. Es decir, si tenemos un récord de producción en el sector minero o energía, y para ello arrojamos relaves a los ríos con destrucción de la flora y fauna acuática, inutilizamos el agua para fines agrícolas, turísticos o de consumo. Si tenemos que gastar más en salud para la recuperación de la población afectada, etcétera, todos estos son costos que no están siendo internalizados a los costos de producción y, por lo tanto, el éxito aparente en la gestión sectorial se convierte en un lastre pesado para el país.

\section{EL MARCO JURÍDICO INSTITUCIONAL AMBIENTAL EN EL PERÚ}

En el Perú se creó en el año 1974 la Oficina Nacional de Evaluación de Recursos Naturales (ONERN), la cual funcionó como punto focal peruano ante el Programa de las Naciones Unidas para el Medio Ambiente. Su función principal estaba vinculada a la realización de inventarios de recursos naturales como recursos hídricos, suelos, flora, fauna, recursos pesqueros, etcétera. La gestión de los recursos estaba asignada fundamentalmente a sectores productivos como agricultura, pesquería, minería, sectores que carecían de un marco normativo adecuado, personal capacitado y recursos financieros. En estos sectores había una clara voluntad política de hacer prevalecer volúmenes de producción antes que cumplir exigencias ambientales. Los aspectos de saneamiento y desarrollo urbano se encontraban en los sectores de salud y vivienda respectivamente, mientras que la planificación en manos del Instituto Nacional de Planificación (INP).

Es a partir del año 1990, con la dación del Código del Medio Ambiente y los Recursos Naturales, que se crea el Consejo Nacional del 
Ambiente (CONAM). La propuesta inicial fue crear un organismo de carácter suprasectorial que se ubicara en el seno de la Presidencia del Consejo de Ministros a efectos de evitar conflictos entre sectores, contando con un Consejo Directivo donde estuvieran representados además de representantes del Poder Ejecutivo, representantes de los sectores productivos y sociales. El CONAM debía ser un espacio donde se adoptara la política nacional del ambiente, la cual debía ser de obligatorio cumplimiento para todos los sectores. Rápidamente se desnaturalizó el CONAM, convirtiéndose en un ente ejecutor, pero sin la fortaleza necesaria y su consejo directivo fue copado por el sector empresarial.

El año 2008, mediante decreto legislativo 1013, se produjo la creación del Ministerio del Ambiente. Fue una decisión política rápida en el marco de la negociación de un tratado de libre comercio, hecho que género el nacimiento de un organismo sin una visión clara respecto a su rol en la gestión ambiental. No queda claro si se pretendió crear un ministerio aglutinador de todas las competencias ambientales o uno que integre determinadas funciones. Lo cierto es que el Ministerio del Ambiente en la actualidad ha logrado asumir competencias ambientales específicas, pero a su vez coexiste con estructuras jurídico administrativas preexistentes en sectores productivos como minería, industrias, pesca, hidrocarburos, etcétera.

La creación del Ministerio del Ambiente, a partir de un tránsito de competencias ambientales sectoriales, y la creación de un Consejo Nacional del Ambiente (CONAM), fenómeno ocurrido en casi toda América Latina y el Caribe ${ }^{13}$, no evidencian una mejora sustancial en la gestión ambiental del país. Luego de su creación apareció con nitidez una carencia enorme en los gobernantes de turno en relación con cuál era el rol de este nuevo ministerio y cómo debía interactuar con otros sectores como economía y fundamentalmente con los sectores productivos como minería, energía, pesquería, industrias, agricultura, etcétera. En la actualidad, vemos disputas muy claras al interior del Ejecutivo respecto a las competencias que aún mantienen sectores productivos. La creación de un sistema nacional de certificación ambiental no es otra cosa que la evidencia de esas disputas fundamentalmente con el sector minero energético. Además, en vez de crear mecanismos y procedimientos claros y transparentes que fortalezcan la institucionalidad ambiental, vemos ministerios disputando competencias, lo cual evidencia la carencia de una institucionalidad ambiental fuerte y con competencias claramente definidas. Uno de los grandes temas es la aprobación de los estudios de impacto ambiental (EIA), sin embargo, el tema de la fiscalización respecto al cumplimiento de los compromisos contenidos en los EIA 
también es tarea fundamental como lo son los planes de cierre de las actividades productivas. Qué hacer con los pasivos ambientales es un grave problema no resuelto.

La creación de la Oficina de Evaluación y Fiscalización Ambiental (OEFA) podría ayudar mucho en el proceso de fortalecimiento de la gestión ambiental. Sin embargo, ello requiere, desde nuestro punto de vista, adicionalmente a la dotación de recursos económicos, técnicos y humanos, dotarla de una independencia que la haga ajena a la presión del gobierno de turno. El jefe de esa entidad debiera ser designado por el Congreso de la República, pues el Poder Ejecutivo es el principal favorecido con la renta que producen las actividades extractivas. Un claro ejemplo lo constituye el sector minero que aporta más del sesenta por ciento de los ingresos del país y que difícilmente va a ser fiscalizado por el Poder Ejecutivo con la debida rigurosidad. Asimismo, los miembros del tribunal de fiscalización ambiental debieran ser designados por el Consejo Nacional de la Magistratura y sus miembros no debieran estar vinculados a gremios empresariales o ser parte de instituciones con intereses contrapuestos a la fiscalización ambiental como ocurre en la actualidad.

Respecto a los funcionarios con competencias ambientales en los sectores productivos, estos deberían ser designados por el jefe de la OEFA, en tanto este fuera designado fuera del ámbito del Poder Ejecutivo. Además, no deberían poder ser removidos de sus cargos por los titulares de los sectores sin su anuencia si considera que están cumpliendo a cabalidad sus funciones fiscalizadoras. Este sistema, consideramos, podría ayudar muchísimo a integrar e internalizar la dimensión ambiental en las actividades productivas y posibilitar la creación de una disciplina ambiental. Además, ayudaría a lograr una continuidad e interdependencia de acción que permitiría instaurar políticas a mediano y largo plazo. Actualmente la burocracia ambiental no tiene, jurídicamente, elementos suficientes para cerrarle el paso a decisiones políticas ligadas en muchos casos a grupos económicos de mucho poder que operan con recursos naturales. Adicionalmente, si se garantiza la permanencia de la burocracia ambiental, se podrían iniciar programas de capacitación hasta contar con personal con conocimientos especializados, inicial y subsecuentemente, es decir, personal actualizado con pleno conocimiento de los ecosistemas nacionales.

Otro aspecto que le quita mucha fuerza al Ministerio del Ambiente es la carencia de recursos económicos. Desde su creación, el Ministerio del Ambiente se ha convertido en un centro de imputación frente a la agudización de los problemas ambientales, muchos de ellos han devenido en graves conflictos sociales como lo fue el caso de Bagua y otros problemas como los de Espinar y Conga. Pero poco ayuda 
una institución carente de recursos de toda índole, el año 2012, el presupuesto del Ministerio del Ambiente no llegaba a los 250 millones de soles, eso equivalía a poco más del dos por ciento de lo recaudado por el Estado peruano únicamente por concepto de impuesto a la renta proveniente de la actividad minera, una actividad no solamente con altos impactos ambientales actuales, sino que arrastra en su haber grandes pasivos ambientales. Aquí les corresponde a los empresarios confrontar al Estado a que también cumpla su responsabilidad, pues no solo debe haber un Estado ávido de recaudar más impuestos, sino que tiene que invertir parte de lo recaudado en remediar los impactos ambientales que le produce generar esa renta. A manera de ejemplo podemos referirnos a lo que ocurre en la provincia cajamarquina de Hualgayoc, donde el Estado reconoce la existencia de más de mil doscientos pasivos ambientales mineros cuya remediación bordea los setenta millones de soles y, sin embargo, no hace gran cosa por este problema específico. Si el Estado invirtiera en la remediación de pasivos ambientales, generaría un clima amigable con las comunidades que posibilitaría el desarrollo de futuros proyectos, como es el caso de Conga ubicado precisamente en Hualgayoc.

Particularmente consideramos que si el esquema institucional no cambia, los conflictos ambientales se van a agudizar. Consideramos que seguir aglutinando competencias en el Ministerio del Ambiente, sin la provisión de los recursos adecuados, no solo debilita los avances en el fortalecimiento alcanzado en algunos sectores productivos en materia ambiental, sino que crea la convicción errónea de que los problemas ambientales están siendo debidamente afrontados. Se debe ponderar la real capacidad de gestión del Ministerio del Ambiente antes de tratar de hacer de él el superministerio al que se integran todas las competencias ambientales sectoriales.

Tampoco se debe perder de vista que además de las competencias ambientales que concentra el Ministerio del Ambiente y mantienen diversos sectores del Gobierno Central, de conformidad con lo establecido en la Constitución Política del Estado, los gobiernos regionales y locales tienen una serie de competencias respecto al uso de los recursos naturales ubicados en sus jurisdicciones así como el cuidado de los elementos ambientales. Los gobiernos regionales y locales han asumido diversas responsabilidades en materia de medio ambiente y recursos naturales que requieren contar con una estructura administrativa de la cual carecen actualmente. Su implementación es urgente para evitar onerosos costos económicos futuros y el aniquilamiento de sus posibilidades de desarrollo.

Los gobiernos locales no han logrado consolidarse como tales. En materia ambiental no se encuentran en condiciones de desempeñar 
a cabalidad las funciones que le asigna la Ley Orgánica de Gobiernos Locales, debido principalmente a una falta de conciencia de la estrecha relación entre ambiente y desarrollo y a la carencia de recursos humanos y financieros. Consideramos que el trabajo a desarrollar con estos niveles de gobierno es prioritario si tenemos en cuenta que estos, como primeros ámbitos de organización política y territorial, pueden servir como catalizadores de la confluencia de voluntades políticas respecto del ambiente inmediato, deseado y posible de la comunidad. Es en el ámbito del gobierno local donde la sociedad puede conocer mejor su realidad ambiental, principalmente por su proximidad con el medio natural y con las actividades humanas que se desarrollan en su jurisdicción. 


\begin{tabular}{|c|c|}
\hline País & Institución ambiental \\
\hline Antigua y Barbuda & $\begin{array}{l}\text { Ministry of Agriculture, Lands, Housing and } \\
\text { the Environment }\end{array}$ \\
\hline Argentina & $\begin{array}{l}\text { Secretaría de Medio Ambiente y Desarrollo } \\
\text { Sustentable de la Nación }\end{array}$ \\
\hline Bahamas & Ministry of Environment \\
\hline Estado Plurinacional de Bolivia & Ministerio de Medio Ambiente y Agua \\
\hline Brasil & Ministerio de Medio Ambiente \\
\hline Chile & Ministerio de Medio Ambiente \\
\hline Colombia & $\begin{array}{l}\text { Ministerio de Ambiente y Desarrollo } \\
\text { Sostenible }\end{array}$ \\
\hline Costa Rica & Ministerio de Ambiente, Energía y Mares \\
\hline Cuba & $\begin{array}{l}\text { Ministerio de Ciencia, Tecnología y Medio } \\
\text { Ambiente }\end{array}$ \\
\hline Dominica & $\begin{array}{l}\text { Ministry for Environment, Natural } \\
\text { Resources, Physical Planning and Fisheries }\end{array}$ \\
\hline Ecuador & Ministerio del Ambiente \\
\hline El Salvador & $\begin{array}{l}\text { Ministerio de Medio Ambiente y Recursos } \\
\text { Naturales }\end{array}$ \\
\hline Grenada & $\begin{array}{l}\text { Ministry of the Environment, Foreign Trade } \\
\text { and Export Development }\end{array}$ \\
\hline Guatemala & $\begin{array}{l}\text { Ministerio de Ambiente y Recursos } \\
\text { Naturales }\end{array}$ \\
\hline Guyana & $\begin{array}{l}\text { Ministry of Natural Resources and the } \\
\text { Environment }\end{array}$ \\
\hline Haití & Ministry of Environment \\
\hline Honduras & $\begin{array}{l}\text { Secretaría de Recursos Naturales y } \\
\text { Ambiente }\end{array}$ \\
\hline
\end{tabular}

MARCO

INSTITUCIONAL PARA LA GESTIÓN AMBIENTAL EN EL PERÚ

INSTITUTIONAL FRAMEWORK FOR ENVIRONMENTAL MANAGEMENT IN PERU 


\begin{tabular}{|c|c|}
\hline País & Institución ambiental \\
\hline Jamaica & $\begin{array}{l}\text { Ministry of Water, Land, Environment and } \\
\text { Climate Change }\end{array}$ \\
\hline México & $\begin{array}{l}\text { Secretaría de Medio Ambiente y Recursos } \\
\text { Naturales }\end{array}$ \\
\hline Nicaragua & $\begin{array}{l}\text { Ministerio del Ambiente y Recursos } \\
\text { Naturales }\end{array}$ \\
\hline Panamá & Autoridad Nacional del Ambiente \\
\hline Paraguay & Secretaría del Ambiente \\
\hline Perú & Ministerio del Ambiente \\
\hline República Dominicana & $\begin{array}{l}\text { Ministerio de Ambiente y Recursos } \\
\text { Naturales }\end{array}$ \\
\hline St. Kitts and Nevis & Ministry of Sustainable Development \\
\hline St. Lucia & $\begin{array}{l}\text { Ministry of Public Service, Sustainable } \\
\text { Development, Energy, Science and } \\
\text { Technology }\end{array}$ \\
\hline St. Vincent and the Grenadines & $\begin{array}{l}\text { Ministry of Health, Wellness and the } \\
\text { Environment }\end{array}$ \\
\hline Suriname & $\begin{array}{l}\text { Ministry of Labour, Technological } \\
\text { Development and Environment }\end{array}$ \\
\hline Trinidad y Tobago & Ministry of Housing and the Environment \\
\hline Uruguay & $\begin{array}{l}\text { Ministerio de Vivienda, Ordenamiento } \\
\text { Territorial y Medio Ambiente }\end{array}$ \\
\hline $\begin{array}{l}\text { República Bolivariana de } \\
\text { Venezuela }\end{array}$ & $\begin{array}{l}\text { Ministerio del Poder Popular para el } \\
\text { Ambiente de Venezuela }\end{array}$ \\
\hline
\end{tabular}

